\title{
The molecular emission from old supernova remnants
}

\author{
A. Gusdorf ${ }^{1}$, R. Güsten ${ }^{2}$, S. Anderl ${ }^{3}$, T. Hezareh ${ }^{2}$, \\ and H. Wiesemeyer ${ }^{2}$ \\ ${ }^{1}$ LERMA, UMR 8112 du CNRS, Observatoire de Paris, École Normale Supérieure, \\ 24 rue Lhomond, F75231 Paris Cedex 05, France, email: antoine.gusdorf@lra.ens.fr \\ ${ }^{2}$ Max Planck Institut für Radioastronomie, Auf dem Hügel 69, 53121 Bonn, Germany \\ ${ }^{3}$ Argelander Institut für Astronomie, Universität Bonn, Auf dem Hügel 71, 53121 Bonn, \\ Germany
}

\begin{abstract}
Supernovae constitute a critical source of energy input to the interstellar medium (ISM). In this short review, we focus on their latest phase of evolution, the supernova remnants (SNRs). We present observations of three old SNRs that have reached the phase where they interact with the ambient interstellar medium: W28, IC443, and 3C391. We show that such objects make up clean laboratories to constrain the physical and chemical processes at work in molecular shock environments. Our studies subsequently allow us to quantify the impact of SNRs on their environment in terms of mass, momentum, and energy dissipation. In turn, their contribution to the energy balance of galaxies can be assessed. Their potential to trigger a further generation of star formation can also be investigated. Finally, our studies provide strong support for the interpretation of $\gamma$-ray emission in SNRs, a crucial step to answer questions related to cosmic rays population and acceleration.
\end{abstract}

Keywords. ISM: supernova remnants - Shock waves - Submillimeter: ISM - ISM: individual objects: W28, IC443, \& 3C391 - Stars: formation - cosmic rays.

\section{The astrophysical importance of supernova remnants}

The life of massive stars ends with a supernova explosion, characterized by an important redistribution of energy towards the interstellar medium (ISM). After a free expansion phase (ending when the swept-up mass reaches that of the envelope), and an adiabatic phase (where the energy dissipation is due to expansion), the supernovadriven shocks start radiating energy (Woltjer 1972), initially at observable optical and ultraviolet wavelengths from what has become supernova remnants (SNRs; e.g. Weiler \& Sramek 1988). When SNRs encounter molecular clouds, they drive slower shock waves that compress, accelerate and heat the molecular material and result in strong infrared and sub-millimeter line emission (e.g. Neufeld et al. 2007, Frail \& Mitchell 1998).

These relatively slow molecular shocks are found to be very similar to those observed in the jets and outflows associated with star formation (e.g. Gusdorf et al. 2011). However, contrary to their star-formation counterparts, SNR shocks are not expected to be contaminated by the possible UV radiation from the proto-star. Additionally, spectral lines observed in SNR shock regions do not either show any envelope or infall component (see for instance the upper right panel in Fig. 1) that would make their interpretation complicated. SNRs hence serve as clean laboratories to study the physical and chemical mechanisms that operate in shock environments.

In a further step, studying SNRs interacting with the ISM is a powerful tool to quantify their contribution to the energy balance of galaxies, through the observation of the numerous CO transitions recently allowed by the Herschel telescope, and presented in 

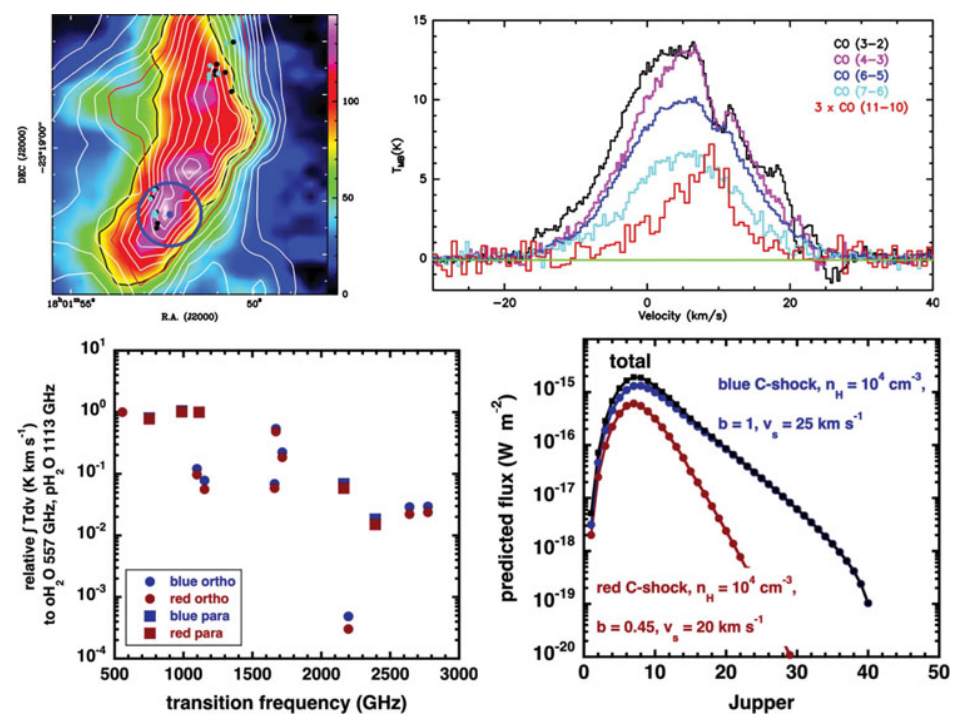

Figure 1. CO observations of W28, as presented in Gusdorf et al. (2012). Top, left: the field covered by our maps of the CO (6-5) and (3-2) transitions (colours, white contours). The small dots are the $\mathrm{OH}$ masers (Claussen et al. 1997 and Hoffman et al. 2005). Our shock analysis was made on the position at the center of the circle, that indicates the beam of our SOFIA observations. Top, right: the CO spectra (obtained with APEX, (3-2), (4-3), (6-5) and (7-6), and SOFIA, (11-10)), convolved to the SOFIA resolution, were extracted from this position. They were combined with $\mathrm{H}_{2}$ Spitzer observations (Neufeld et al. 2007) to constrain our shock models. Bottom, left: the models can subsequently be used to make water emission predictions. Bottom, right: they can also be used to predict the emission of all of the CO transitions.

flux diagrams, the so-called CO ladders (e.g. Hailey-Dunsheath et al. 2012). In certain cases, their potential to trigger star formation can be investigated, see $\mathrm{Xu}$ et al. (2011).

Finally, the molecular emission from SNRs provide a valuable support for the study of cosmic rays population (CRs, hadronic or leptonic) and acceleration. Because of their large energy budget, it has indeed long been argued that SNRs are the primary sites for accelerating CRs (e.g., Blandford \& Eichler 1987). Observing the $\gamma$-ray emission in SNRs environments is a way to constrain the CRs population, and acceleration mechanisms. Indeed, this radiation mainly results from three processes: $\pi^{0}$ decay from the interaction of the hadronic component of the CRs population with the ambient medium, Bremsstrahlung emission from the leptonic component on the ambient ISM, and inverse Compton scattering of the leptonic component on the ambient radiation field, (e.g. Frail 2011 for a short review). Although the situation is a bit more complex for old SNRs (e.g., Gabici et al. 2009, Bykov et al. 2000), whose shocks no longer accelerate CRs, an accurate knowledge of the ambient medium (density, mass of the shocked/non shocked gas, local magnetic field strength, local radiation field) remains crucial to assess the contribution from each of these three processes to the very high energy spectra observed in SNRs.

We demonstrate how the observation of the molecular emission from three old SNRs, W28, 3C391, and IC443, allow to address these diverse astrophysical topics.

\section{Shock modelling in W28F}

The upper panels of Figure 1 summarize the results presented in Gusdorf et al. (2012), dedicated to observations and models of shocked regions in the W28 SNR. By analysing the $\mathrm{CO}$ (from APEX and SOFIA telescopes) and $\mathrm{H}_{2}$ (from the Spitzer telescope) 

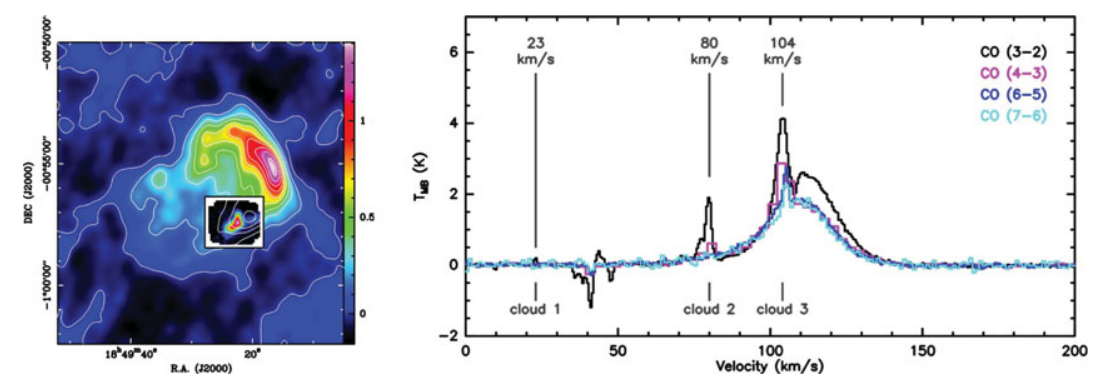

Figure 2. Left: $325 \mathrm{MHz}$ radio-continuum emission in the 3C391 SNR, as it appeared in Moffett \& Reynolds (1994) (colours and white contours). The small inset is the field of our CO observations with the APEX telescope, and shows the CO (6-5) emission. Right: the spectra in various CO lines observed with the APEX telescope, averaged over the whole observed field.

emission, we have constrained shock parameters in the beam of our observations. For this position, we have extracted two CO integrated intensity diagrams, one per velocity range (blue lobe, -30 to $\sim 10 \mathrm{~km} \mathrm{~s}^{-1}$, and red lobe, $\sim 10$ to $40 \mathrm{~km} \mathrm{~s}^{-1}$ ). We have compared the corresponding high- $J$ CO emission, that most unambiguously traces the shocked material, to a grid of unidimensional shock models. We hence have fitted each velocity component by a single, C-type shock wave arising from a $25^{\prime \prime}$ diameter emission region, with the respective parameters: pre-shock density $n_{\mathrm{H}}=10^{4} \mathrm{~cm}^{-3}$, magnetic field strength perpendicular to the shock front $B=100$ and $45 \mu \mathrm{G}$, and shock velocities $v_{\mathrm{s}}$ $=20$ and $25 \mathrm{~km} \mathrm{~s}^{-1}$. We also checked that our models provide reasonable fits for $\mathrm{H}_{2}$ excitation diagrams (Spitzer observations by Neufeld et al. 2007). Our final results are compatible with independent studies of the region in terms of age (Giuliani et al. 2010), magnetic field measurements (Claussen et al. 1997, Hoffman et al. 2005), and densities required for the excitation of observed $\mathrm{OH}$ masers (Lockett et al. 1999).

In a forthcoming publication (Gusdorf et al., in prep), we will go one step further in the interpretation of the results. With the shock model parameters, and assumptions on the size of the emitting region, we can thus infer the shocked mass in the beam of our observations (from 6.2 to $18.5 M_{\odot}$ depending on the adopted age, $10^{4}$ or $3 \times 10^{4}$ years, respectively). Combining our shock model results with an LVG code to calculate the emission from water (Gusdorf et al. 2011), we are also able to predict integrated intensity diagrams for each velocity component, which can be compared for instance to observations made by the Herschel telescope, as can be seen on the lower left panel of the Figure 1. Additionally, we predict the excitation from all CO lines in our beam (lower right panel of the Figure 1), that can be directly compared with Herschel observations of the region. Such SNR shocks CO ladders can also directly be compared an help constraining their contribution to galactic ones (e.g. Hailey-Dunsheath et al. 2012).

\section{The cases of $3 \mathrm{C} 391$ and IC443}

Forthcoming publications will also be focused on a similar analysis of SNRs 3C391 and IC443 (Gusdorf et al., in prep), based on $\mathrm{CO}$ and $\mathrm{H}_{2}$ observations.

In 3C391, we find that our APEX CO observations directly allow to identify the lineof-sight clouds that lie in the vicinity of the remnant, and constitute potential targets for the cosmic rays interactions. Figure 2 shows both the field of our observations (left panel), and the averaged CO spectra we extracted (right panel). Based on our CO and ${ }^{13} \mathrm{CO}$ observations, we were able to infer the mass of the clouds that might be impacted 


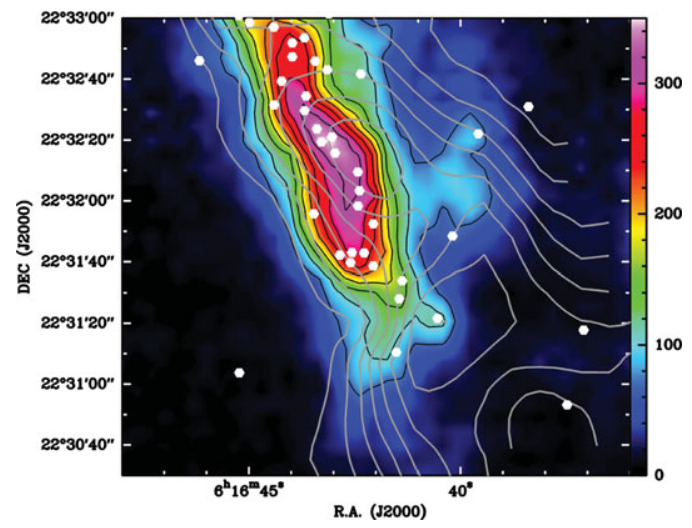

Figure 3. The G clump of the IC443 SNR, mapped with APEX in the CO (6-5) transition (in colours and black contours, Gusdorf et al., in prep). The white markers indicate the position of YSO candidates in the region, selected in the 2MASS point source catalog, based on colour selection criterion by $\mathrm{Xu}$ et al. (2011). Also shown in the greyscale contour is the CO (1-0) ambient emission at $5 \mathrm{~km} \mathrm{~s}^{-1}$, observed by the IRAM $30 \mathrm{~m}$ telescope (Hezareh et al., in prep).

by the cosmic rays accelerated in the SNR, the so-called clouds 2 and 3, respectively 161.3 and $43.7 M_{\odot}$ - see for instance Xu et al. (2011) for the calculation method.

Finally, in the G clump of the IC443 SNR, our observations (Gusdorf et al., in prep, and Hezareh et al., in prep, and Figure 3) confirm the results obtained by Xu et al. (2011). As the YSO candidates in the region are older than the SNR, these authors show that in spite of the correlation between their distribution and the shock structure, star formation has probably not been triggered by the supernova-driven shock wave, but rather by the stellar winds of the massive progenitor of the remnant. Paradoxically, our observations confirm the tight correlation between the star formation and the shocked $\mathrm{CO}(6-5)$ gas in the region. On the other hand, our CO (1-0) observations show that the star formation has developed on the edge of a nearby ambient cloud (also see Lee et al. 2012), subject to the stellar winds of the SNR progenitor prior to the passage of the shock wave.

\section{References}

Blandford, R. \& Eichler, D. 1987, PR, 154, 1

Bykov, A. M., Chevalier, R. A., Ellison, D. C., \& Uvarov, Y. A. 2000, ApJ, 538, 203

Claussen, M. J., Frail, D. A., Goss, W. M., \& Gaume, R. A. 1997, ApJ, 489, 143

Frail, D. A. \& Mitchell, G. F. 1998, ApJ, 508, 690

Frail, D. A. 2011, MemSAIt, 82, 703

Gabici, S., Aharonian, F. A., \& Casanova, S. 2009, MNRAS, 396, 1629

Giuliani, A., Tavani, M., Bulgarelli, A., et al. 2010, A\&SA, 516, L11

Gusdorf, A., Giannini, T., Flower, D. R., et al. 2011, A\&A, 532, A53

Gusdorf, A., Anderl, S., Güsten, R., et al. 2012, A\&A, 542, L19

Hailey-Dunsheath, S., Sturm, E., Fischer, J., et al. 2012, ApJ, 755, 57

Hoffman, I. M., Goss, W. M., Brogan, C. L., \& Claussen, M. J. 2005, ApJ, 620, 257

Lee, J.-J., Koo, b.-C., Snell, R. L., et al. 2012, ApJ, 749, 34

Lockett, P., Gauthier, E., \& Elitzur, M. 1999, ApJ, 511, 235

Moffett, D. A. \& Reynolds, S. P. 1994, ApJ, 425, 668

Neufeld, D. A., Hollenbach, D. J., Kaufman, M. J., et al. 2007, ApJ, 664, 890

Weiler, K. W. \& Sramek, R. A. 1988, ARAA, 26, 295

Woltjer, L. 1972, ARAA, 10, 129

Xu, J.-L., Wang, J.-J., \& Miller, M. 2011, ApJ, 727, 81 


\section{Discussion}

UNIDENTIFIED: I am curious as to how you determined the shock velocity as an input for the model. This is quite interesting because determining the shock velocity from a numerical simulation is not easy due to shock broadening.

GusDorf: A range of possible shock velocities arise from the observations, that provide an upper limit to them. The rest is determined like the other input parameters, through a comparison of all lines observed to our models with a $\chi^{2}$ method. Some theoretical considerations also exist that constrain the shock velocity range for a given set of (other) shock parameters ("critical velocities").

Zhоu P.: What is the temperature of the MC-F in W28?

GuSDORF: There are several temperature components along the line of sight/ shocked layer. The one that is probed by the $\mathrm{CO}$ observations presented here is typically $\sim 100 \mathrm{~K}$, but higher lines can be excited. A strong point in our models is that we expect all these components to be accounted for, unlike in the case of a single-slab LVG model, for instance. 